https://artnodes.uoc.edu

\title{
El autor y el intérprete: una mirada contemporánea al proyecto del Biocentro para la Universidad de Frankfurt de Peter Eisenman
}

\author{
Marcelo Fraile-Narváez \\ Escuela Superior de Ingeniería y Tecnología \\ Universidad Internacional de La Rioja
}

Fecha de presentación: mayo de 2021

Fecha de aceptación: enero de 2022

Fecha de publicación: enero de 2022

\section{Cita recomendada}

Fraile-Narváez, Marcelo. 2022. «El autor y el intérprete: una mirada contemporánea al proyecto del Biocentro para la Universidad de Frankfurt de Peter Eisenman». Artnodes, no. 29. UOC. [Fecha de consulta: dd/mm/aa]. https://doi.org/10.7238/artnodes.v0i29.387828 \footnotetext{
$\begin{array}{cl}\text { CC. } & \begin{array}{l}\text { Los textos publicados en esta revista están sujetos -si no se indica lo contrario- a una licencia } \\ \text { de Reconocimiento } 4.0 \text { Internacional de Creative Commons. La licencia completa se puede consultar } \\ \text { en https://creativecommons. org/licenses/by/4.0/deed.es }\end{array}\end{array}$
}

\section{Resumen}

El proyecto para el Biocentro de la Universidad de Frankfurt constituye uno de los proyectos más relevantes de la arquitectura contemporánea. Tomado como referente por muchos investigadores de la talla de Charles Jencks 0 Greg Lynn, algunos lo consideran una prueba tangible del nacimiento de un nuevo paradigma dentro de la arquitectura. Un proyecto que, aunque no construido, ha originado una cantidad proporcional tanto de defensores como de detractores, y ha dejado en el camino que sus ideas influyeran a diversas generaciones de diseñadores.

$Y$ es precisamente en este sentido, y con la suficiente perspectiva histórica para ello, donde se hace necesaria una revisión exhaustiva de los procesos y de los actores que dieron vida al proyecto del biocentro. Un proceso complejo que, a priori, involucraba dos mundos tan contrastantes como lo son el analógico, representado por Peter Eisenman, y el digital, representado por Chris Yessios.

A partir de estos planteamientos, este trabajo tiene como objetivo principal desentrañar la relevancia de Yessios durante el proceso proyectual que condujo al diseño del biocentro. A través de una investiga- 
ción arqueológica de la arquitectura generativa, se propone demostrar que la actuación de Yessios parece superar la de mero programador informático, para transformarse, al desarrollar las herramientas y los procesos que posibilitaron su ejecución, en intérprete, pero también en coautor del proyecto.

Se busca identificar la complejidad del programa proyectual, describiendo y analizando la actuación de su autor durante el proceso de gestación de la obra, y la de su intérprete, como ejecutor de los procesos de trabajo, buscando reconocer y comprender sus propias aportaciones al propio sentido de dicha tarea.

Finalmente, este ensayo no pretende cerrar el debate, sino aportar una nueva.

\title{
Palabras clave
}

Eisenman; Yessios; Biocentro de Frankfurt; geometría fractal; arquitectura generativa; diseño generativo

\section{The author and the interpreter: a contemporary look at Peter Eisenman's Biocenter project for the University of Frankfurt}

\begin{abstract}
The project for the Biocenter of the University of Frankfurt is one of the most relevant in contemporary architecture. Considered a benchmark by many researchers of the stature of Charles Jencks or Greg Lynn, some of them view it as tangible proof of the birth of a new paradigm in architecture. This project, although not yet built, gave rise to a proportional number of both defenders and detractors, while influencing several generations of designers along the way.

It is precisely in this sense and considering it with the necessary historical perspective, that an exhaustive review of the processes and actors that gave life to the Biocenter project seems necessary. A complex process that a priori involved two contrasting worlds: the analogical, represented by Peter Eisenman, and the digital, by Chris Yessios.

From these approaches, the main objective of this work is to unravel the relevance of Yessios' participation during the design process that led to the design of the Biocenter. Through archaeological research of generative architecture, it is possible to demonstrate that Yessios' performance seems to go beyond that of a mere computer programmer, to become - by developing the tools and processes that made its execution possible - an interpreter, as well as a co-author of the project.

A search that will aim to identify the complexity of the project programme, describing and analyzing the performance of its author during its gestational period, and that of its interpreter, as executor of the work processes, seeking to identify and understand his own contributions to the very meaning of this task.

Finally, this essay does not intend to close the debate, but, on the contrary, to provide a new perspective that encourages the creation of future works related to the subject: a sample of the existing complexity within the field of design.
\end{abstract}

\section{Keywords}

Eisenman; Yessios; Frankfurt Biocenter; fractal geometry; generative architecture; generative design 


\section{Introducción: el Biocentro para la Universidad de Frankfurt, Alemania}

En 1987, el arquitecto americano Peter Eisenman fue invitado a participar en el Concurso Internacional para el desarrollo de un Centro de Investigaciones Biológicas Avanzadas, organizado por la Universidad de Frankfurt, en Alemania. Conocido como biocentro, la competencia preveía la ampliación de los laboratorios de investigación de biotecnología, biología molecular y bioquímica, y espacios anexos de apoyo a los edificios principales existentes en el campus.

Dos hechos fortuitos se conjugarían en el desarrollo de este proyecto que llevarían a Eisenman a ser galardonado con un premio especial. El primero de ellos debemos atribuirlo al deseo del autor de distanciarse de los criterios normativos de la arquitectura tradicional, buscando explorar nuevos rumbos que lo llevaran a traspasar los límites disciplinares. Por aquel entonces, Eisenman se encontraba muy interesado en las teorías sobre el lenguaje desarrolladas por el filósofo y lingüista estadounidense Noam Chomsky, que proponía lo que él llamaba «gramática generativa»: un proceso innato del cerebro humano a través del cual, este era capaz de traducir una combinación determinada de ideas, en una composición de códigos (1974).

El segundo de estos hechos fortuitos se debió a una extraña coincidencia espaciotemporal. A principios de la década de 1980, Eisenman era profesor invitado del Departamento de Arquitectura de la Universidad Estatal de Ohio (OSU), donde conocería a Chris Yessios ${ }^{1}$ y a Jerry Voss, ${ }^{2}$ famosos por promover la utilización de los ordenadores en la investigación y el diseño.

En 1982, Eisenman y Yessios, trabajarían juntos por primera vez en el proyecto para el Wexner Center for the Visual Arts and Fine Arts Library, en el que comenzarían a experimentar con el uso del ordenador dentro del entorno del diseño. ${ }^{3}$ Desde este punto de vista, Yessios resultaría ser una figura clave en la búsqueda de una herramienta efectiva para la construcción de los diseños planteados por Eisenman en el futuro. Y es precisamente en este sentido, y con la suficiente perspectiva histórica para ello, donde se hace necesaria una revisión exhaustiva de los procesos y de los actores, que dieron vida al proyecto del Biocentro. Un proceso complejo que, a priori, involucraba dos mundos tan contrastantes como lo eran el analógico, representado por Peter Eisenman, y el digital, por Chris Yessios.
En el proyecto del Biocentro se revela «una estructura profunda cuyas leyes gobiernan el desarrollo de la obra: su proceso de gestación, sus códigos, prevalecen en el objeto y quedan plasmados en él» (García Sánchez 2018, 89). Sin embargo, el problema resulta mucho más complejo aún: sus diagramas y axonométricas «revelan que en el proceso no se tuvo en consideración si el resultado era arquitectura [...] el problema no fue diseñar un objeto, sino buscar y establecer un programa transformacional libre de las constantes autoriales tradicionales» (García Sánchez 2018, 91).

A partir de estos planteamientos, este trabajo tiene como objetivo principal desentrañar la relevancia de Yessios durante el proceso proyectual que condujo al diseño del Biocentro de Eisenman. Una investigación arqueológica que intentará identificar la complejidad del programa proyectual, describiendo y analizando la actuación de su autor en la gestación de la obra, y la de su intérprete, como ejecutor de los procesos de trabajo, buscando reconocer y comprender sus propias aportaciones proyectuales al sentido mismo de dicha tarea.

Para el arquitecto Rafael Moneo, lo más interesante de este proceso es «que la arquitectura se ha convertido en un objeto cuya forma no es caprichosa dado que ha recogido todas las operaciones geométricas de que da cuenta el proceso» $(2004,158)$, y los ordenadores son utilizados como máquinas conceptuales para generar alternativas proyectuales.

\section{La plataforma digital: ARCHIMODOS}

A principios de la década de 1980, Jerry Voss había firmado un convenio con la empresa IBM, para que la OSU pudiera utilizar para sus investigaciones un ordenador modelo IBM $4341^{4}$ con 16 terminales. Estas estaciones de trabajo contaban con un terminal gráfico rasterizado en color IBM 3279, un teclado, un lápiz óptico, una caja de teclas de funciones y un terminal gráfico de refresco vectorial IBM 3251.

Como parte del acuerdo suscrito entre IBM y la OSU, IBM había entregado 1.000.000 de dólares para que la Universidad desarrollara nuevas investigaciones informáticas que generaran sistemas $C A D$ innovadores. A raíz de este contrato, entre enero de 1982 y marzo de 1985, Yessios desarrollaría un software llamado ARCHIMODOS. ${ }^{5}$ En esencia, se trataba de una plataforma que reunía los estudios producidos durante diez años en los Laboratorios de Diseño Ambiental y

1. Director del posgrado en Diseño Asistido por Ordenador, de la Universidad Estatal de Ohio (1973-1995).

2. Director de la Universidad Estatal de Ohio.

3. Cuestionando las ideas convencionales de contextualismo, Eisenman, utiliza un proceso de superposición y escalamiento para introducir una red de calles de la ciudad sobre la retícula del campus universitario (Jormakka 2017).

4. Pese a que en el libro Archaeology of the Digital, de Greg Lynn (2013) en la página 61 cita al modelo CRAY X-MP como el ordenador utilizado para el proyecto del Biocentro, de acuerdo con el arquitecto Yessios, el modelo utilizado fue el IBM 4341, dato que coincide con el manual de la primera versión de ARCHIMODOS, que fuera utilizado para elaborar el proyecto del Biocentro (Chris Yessios, octubre de 2021)

5. Inicialmente, la primera versión del programa fue denominada «Vitruvius», como homenaje al arquitecto romano de la época de Augusto, tiempo después recibiría el nombre de ARCHIMODOS (ARCHItectural MODeling Design \& Drafting System de la Universidad Estatal de Ohio). 


\section{artnodes}

https://artnodes.uoc.edu

El autor y el intérprete: una mirada contemporánea al proyecto del Biocentro para la Universidad de Frankfurt de Peter Eisenman

Arquitectónico Asistido por Computadora (CAAED Lab) de la Escuela de Arquitectura de la OSU.

El programa fue lanzado oficialmente en mayo de $1985 \mathrm{y}$, aunque había sido concebido para cumplir necesidades educativas, rápidamente pudo satisfacer las demandas de un sistema CAD orientado al diseño profesional. Una de sus cualidades más importantes es que permitía a los usuarios, principalmente arquitectos, alternar las vistas entre 2D y 3D, algo que para Yessios era inherente al proceso proyectual.

En este sentido, Yessios consideraba que la arquitectura no es una extrusión de planos de planta, sino una conceptualización en 3D. De la misma manera, los programas debían ser un instrumento que potenciara esta forma de trabajo, que permitiera crear y explorar las estructuras digitales dentro de un espacio tridimensional.

Para desarrollar este concepto, Yessios había comenzado a experimentar con Void Modeling, una evolución en el pensamiento proyectual que, a diferencia del modelado geométrico de sólidos utilizado en la época, generaba un modelo computable flexible compuesto por dos partes independientes: un elemento contenido (el vacío) y una cáscara (contenedora), cuyos atributos podían ser asignados durante el proceso. A este efecto, el programa contaba con una interfaz ordenada de herramientas que evitaba abrumar al diseñador, lo que posibilitaba el modelado de formas platónicas predefinidas que podían transformarse geométricamente de acuerdo con un conjunto de modificadores.

Inicialmente, al tratarse de un proyecto académico, ARCHIMODOS fue escrito en FORTRAN IV, ${ }^{6}$ codificado para su utilización en ordenadores de grandes dimensiones (Mainframe) que estaban diseñados para el tratamiento de enormes volúmenes de datos. Sin embargo, con la llegada de los años ochenta, y con la proliferación de los ordenadores personales, Yessios optaría por desarrollar el software para su uso en Macintosh, ya que era la única plataforma que admitía gráficos de computadora interactivos con el nivel que se necesitaba en diseño. ${ }^{7}$

\section{Un atípico curso de diseño}

En 1987, Eisenman y Yessios ofrecerían para los semestres de primavera y verano un curso atípico de diseño en el Departamento de Arqui- tectura de la OSU. Para Yessios, se trataba de un curso atípico, ya que a diferencia de las clases tradicionales de diseño en las que el proceso se realizaba mediante una aproximación empírica en etapas sucesivas, este tenía como objetivo utilizar el ordenador durante el proceso proyectual como una herramienta para la exploración y generación de formas arquitectónicas $(1987,171)$. Para esto, además de Eisenman y Yessios, se invitó a formar parte del equipo de profesores a Ben Gianni, Thomas Leeser y Mark Wigley. ${ }^{8}$

Como se trataba de un curso optativo dentro del programa de posgrado de la OSU, se inscribieron nueve estudiantes: siete del $6 .{ }^{\circ}$ curso de diseño y dos de la especialización en diseño arquitectónico asistido por ordenador (CAAD). Al igual que en el estudio de Eisenman, los estudiantes tenían que diseñar un centro de investigaciones biológicas para la Universidad de Frankfurt. De este modo, mientras los alumnos buscaban y trabajaban en sus soluciones de diseño, también lo hacían en la oficina de Eisenman en Nueva York con el mismo programa. Esto ofrecía la oportunidad de un continuo intercambio intelectual entre un entorno profesional, que tenía que preocuparse por las realidades del proyecto y los plazos asociados, y un ámbito académico que podía permitirse experimentar y explorar diferentes alternativas. Con el fin de dar un orden que posibilitara llegar a los tiempos establecidos. El trimestre de primavera se dedicó a una búsqueda exploratoria aplicando transformaciones generativas, mientras que el trimestre de verano se destinó a la producción final del proyecto. ${ }^{9}$

Como punto de partida, Eisenman planteó un proceso generativo basado en tres enfoques diferentes: la geometría fractal, ${ }^{10}$ los ornamentos arabescos y los procesos biológicos. Se buscaba un proceso algorítmico generativo que una vez regulado se «dejara libre», para que fuera capaz de producir el equivalente al diseño propuesto. Como consigna adicional se planteó la restricción de que estos procesos no podían copiarse de forma icónica, sino que debían simularse como mecanismos que condujeran de forma posiblemente abstracta a la generación de un proyecto arquitectónico. ${ }^{11}$

Después de un periodo de búsqueda distendida, el equipo fue convencido por Eisenman de que «el problema no consistía en organizar los espacios y conseguir vínculos adecuados y suficientes» (Yessios 1987), sino más bien en elaborar un «proceso generativo que fuera capaz de

6. El FORTRAN (acrónimo de FORmula TRANslation) fue diseñado en 1954 por el científico computacional estadounidense John Backus como un lenguaje de programación relacionado con la notación matemática normal. Conceptualmente, era un «lenguaje simbólico de tipo general que permitía resolver con facilidad la representación de algoritmos para la solución de problemas científicos en términos de instrucciones al computador» (CELADE 1975, 1). Es decir, se trataba de un lenguaje "Compuesto por proposiciones ordenadas secuencialmente de acuerdo con lo estipulado en el algoritmo de solución del problema. Su ejecución se efectuaba en el mismo orden en que se encontraban, es decir, una a continuación de la otra" (CELADE 1975, 3).

7. En enero de 1990, Yessios, Richard Parent (profesor asociado de gráficos por ordenador en la OSU) y David Kropp (exalumno de Yessios) fundarían AutoDesSys (AUTOmated DESign SYStems). En junio de ese mismo año, se presentaba en el Atlanta Expo Centers, en Atlanta Georgia, FormZ versión gamma 0.9: un programa comercial modelador de propósitos generales (la Z representa la tercera dimensión). Un programa orientado a la producción y la representación espacial que abría nuevas posibilidades al diseño escultórico/espacial a un mayor número de usuarios a bajo costo. Con el fin de evitar errores arrastrados de ARCHIMODOS, el programa fue escrito desde cero en lenguaje de programación C, con algunas partes en C+. Codificada para funcionar bajo Macintosh y con una interfaz intuitiva y fácil de usar, la versión 1 fue lanzada el 14 de febrero de 1991. Esta versión de FormZ incluía herramientas relacionadas con las operaciones booleanas, uno de los puntos fuertes de venta del programa debido a que, en aquel entonces, ningún otro programa contaba con ese tipo de herramientas. Cinco años después, en 1996 salía al mercado la versión de FormZ para Windows (Serraino 2002, 23).

8. Anteriormente, Gianni había trabajado con Eisenman, mientras que Leeser y Wigley trabajaban en ese momento en el estudio de Eisenman.

9. Esta etapa fue realizada utilizando métodos manuales tradicionales en la oficina de Eisenman en Nueva York, donde los estudiantes de la OSU se habían mudado durante el verano.

10.Influenciado por las teorías de Benoît Mandelbrot, desarrolladas en su libro The Fractal Geometry of Nature, publicado en 1982.

11.Para el profesor Arie Graafland, en esta idea Eisenman reunía dos miradas diferentes: por un lado, los conceptos de transcripción y mutación propios del campo de la biología; y, por el otro, las nociones de rastro o marca, propias del campo de la semiótica (1996). 


\section{artnodes}

https://artnodes.uoc.edu

El autor y el intérprete: una mirada contemporánea al proyecto del Biocentro para la Universidad de Frankfurt de Peter Eisenman

crecer hasta el nivel necesario según las necesidades programáticas actuales» (Yessios 1987). Como teórico de la arquitectura, Eisenman buscaba «encontrar las estructuras, las leyes o principios que expliquen la aparición de la forma» (Moneo 2004, 148), para esto, en el proyecto del Biocentro, Eisenman introduce un recurso biológico dentro del proceso de diseño con el fin de generar patrones geométricos que definirían la ubicación, la dimensión y la forma de los elementos.

De un modo aleatorio, ${ }^{12}$ Eisenman tomaría como punto de partida el modelo que utilizan los biólogos para explicar la construcción de proteínas por parte del ADN: cuatro figuras geométricas, que representan a cada uno de los aminoácidos involucrados: pentágono para la citosina $(C)$, cuña para la timina $(T)$, arco para la adenina $(A)$ y cinta para la guanina $(G)$. Además, cada una de estas formas estaba representada con un color específico, y cuyas caras internas tenían un par correspondiente al que se les unía para generar una cadena de doble hebra que reproducía el esquema de la proteína del colágeno, un esquema que también se relacionaba con la resistencia a la tracción de las estructuras naturales como, por ejemplo, las existentes en los huesos (Eisenman 1987).

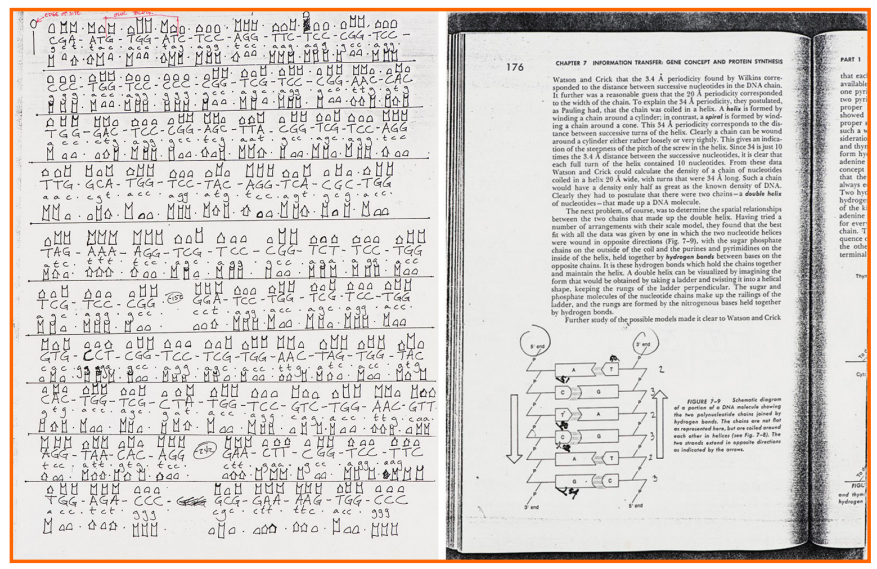

Figura 1 y 2. Representación artificial del ADN, utilizada por los científicos para explicar los procesos genéticos

Fuente: Lynn, Greg. Peter Eisenman, Biozentrum. Montreal: Canadian Centre for Architecture, 2014

Para el proyecto, Eisenman ubicaría sobre el terreno el esquema en escala de la cadena de ADN del colágeno: una secuencia precisa en línea recta que comenzaba en la entrada principal del predio. A continuación, seleccionó los cinco pares de figuras ubicados cerca del edificio de química existente: símbolos tomados del campo de la biología, que se convertirían en los elementos claves para dar origen a los nuevos laboratorios del proyecto (Whiting 2006). Se trataba de una descomposición del objeto en sus partes elementales, para luego aplicarles una serie de operaciones formales. Un proceso generativo análogo al biológico, en el que a partir de los sistemas digitales el proyecto articulaba los tres procesos básicos que se producen en la generación de proteínas: la re- plicación, la transcripción y la traducción. En este sentido, es conveniente destacar que, pese a que Eisenman asegura haber utilizado procesos biológicos, en realidad lo que se utilizaron fueron procedimientos fractales análogos a los procesos biológicos. Es decir, a través del uso de la analogía, los cinco pares de figuras originalmente seleccionados fueron interpretados arquitectónicamente y transformados de manera sucesiva, mediante la utilización de algoritmos digitales, que empleaban técnicas fractales, para generar resultados similares a los procesos biológicos de replicación, transcripción y traducción.

El sistema fractal usado como herramienta de diseño partía de dos elementos: la forma inicial (la forma base) y uno o más generadores (las reglas de producción). Cuando el proceso se iniciaba, el algoritmo iba sustituyendo los segmentos de línea de la forma base seleccionada por nuevos elementos definidos por los generadores en una secuencia de pasos que aumentaba progresivamente su complejidad. Este procedimiento recibía la denominación de «mutación»: una analogía con los procesos existentes en la naturaleza, y donde la forma evolucionaba de acuerdo con los requisitos previamente establecidos.

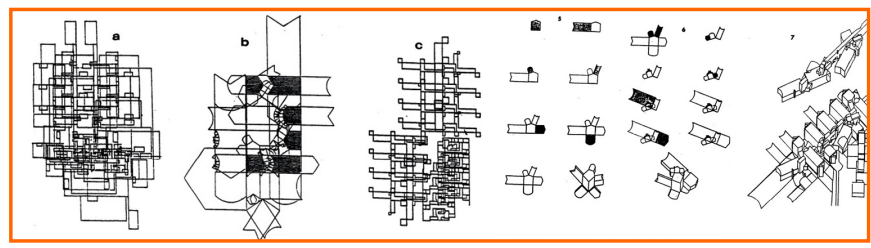

Figuras 3 y 4 . Proceso de diseño utilizando geometrías fractales análogas a los procesos Fuente: Yessios, Chris. «A Fractal Studio». Integrating Computers into the Architectural Curriculum [ACADIA Conference Proceedings] Raleigh (North Carolina / USA): 179-180, 1987

Para que esto sucediera, Yessios y su equipo debieron elaborar una serie de subrutinas que siguieran los sistemas generativos fractales: una sucesión de reglas generadoras, encargadas de reemplazar los elementos iniciales de la forma, y transformarlos en un proceso interactivo de múltiples incrementos. Sin embargo, aunque el sistema permitía sustituir, borrar, insertar o volver a estados anteriores, requería de ciertos ajustes, guías y restricciones por parte de los programadores, ya que, de no hacerse, el sistema continuaría iterando de manera indefinida, sin producir un objeto arquitectónico. Por otro lado, de aplicarse sin ajustar la programación, este procedimiento tenía el potencial de crear formas interesantes, pero carentes de un valor arquitectónico (Yessios 1987, 172). Finalmente, el proceso se completaba con el uso de geometrías arabescas, donde las formas podían superponerse y crecer en diferentes planos, para engendrar una sucesión de patrones repetitivos, que se cruzaban unos sobre otros, produciendo un efecto de entrelazado, que parece cerrarse en sí mismo.

El objetivo de este proceso era utilizar el ordenador como una herramienta de modelado programática, esto es, un sistema capaz de generar figuras en diferentes escalas y posiciones, de acuerdo con una secuencia 


\section{artnodes}

https://artnodes.uoc.edu

El autor y el intérprete: una mirada contemporánea al proyecto del Biocentro para la Universidad de Frankfurt de Peter Eisenman

interminable procedente de una estructura lógica del código, factible de revisarse, adaptarse o repetirse sin cesar. No se trataba de un código de repetición o traslación, sino de una codificación que, reconociendo la posición de los elementos, pudiera cambiar su resultado. El gran reto era emplear técnicas fractales, desarrollar algoritmos que, dependiendo de la escala y la orientación, produjeran resultados distintos (Serraino 2002, 39).

Ahora bien, dado que Eisenman no tenía conocimientos informáticos, el proceso tuvo desde sus inicios una condición mixta, oscilando entre lo digital y lo analógico, algo que muchas veces resultaba engorroso y confuso. Para este proceso, Yessios asumiría el papel de intérprete al traducir a códigos de programación los requisitos de diseño solicitados por Eisenman. Y, aunque existían avanzados sistemas CAD orientados al modelado arquitectónico, ninguno de ellos era lo suficientemente adecuado como para generar los procesos automatizados que Eisenman buscaba. En consecuencia, Yessios tomaría la decisión de utilizar ARCHIMODOS como plataforma base, e ir agregando una variedad de algoritmos generativos que se incorporaban al software, para la creación de formas y elementos en dos y tres dimensiones: un movimiento pendular de carácter vectorial, dentro de un espacio cartesiano de coordenadas y patrones numéricos.

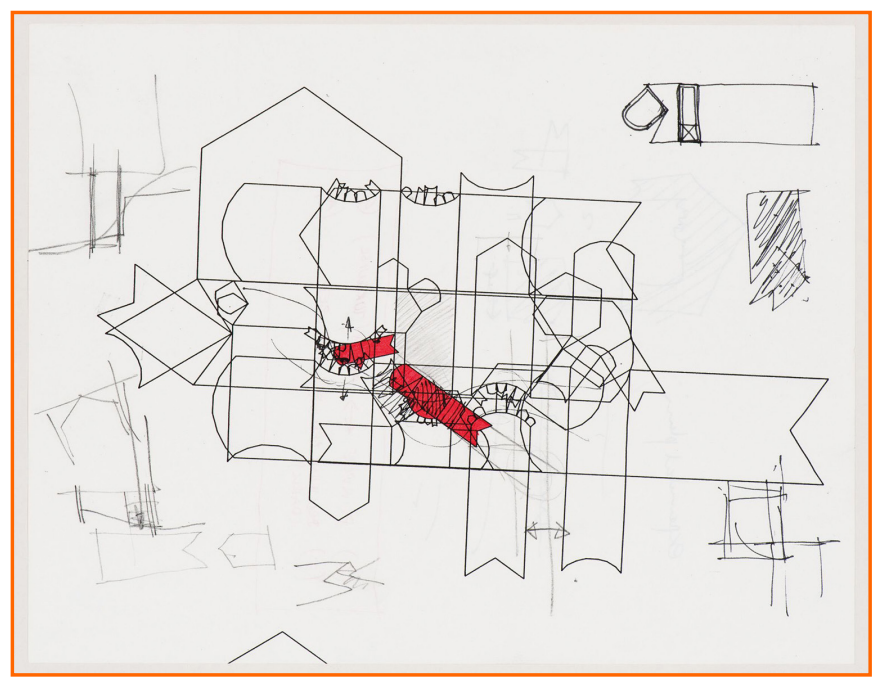

Figura 5. Investigación proyectual para el Centro de Investigaciones Biológicas Avanzadas, de la Universidad de Frankfurt, Alemania

Fuente: Lynn, Greg. Peter Eisenman, Biozentrum. Montreal: Canadian Centre for Architecture, 2014

\section{Fuente: Lynn, Greg. Peter Elsenman, Biozentrum. Montreal Canadian Centre for Architecture, 20}

\section{El proceso de diseño}

El proyecto comenzaba en el estudio de Eisenman, cuando este definía las operaciones formales iniciales. A continuación, Yessios recibía las instrucciones, telefónicamente y por fax, a través de un intrincado código alfanumérico que había sido desarrollado «para agilizar la comunicación» entre ambos nodos: puntos cartesianos y formas geométricas eran escaladas, trasladadas, reflejadas, rotadas y cortadas en un proceso consecutivo. El siguiente paso, ahora a cargo de Yessios, consistía en interpretar y transformar ese código en un lenguaje que pudiera ser introducido en el ordenador, para que este los convirtiera en formas geométricas espaciales: un complicado procedimiento que ante un nuevo pedido de Eisenman, requería reescribir los códigos de programación utilizados.

Con el fin de aligerar el procesamiento de variables, y aprovechando la estructura de 80 líneas que ofrecía FORTRAN IV, Yessios había creado los códigos de programación como diferentes capas de información, permitiendo ajustar las subrutinas para que los resultados pudieran superponerse a modo de layers, y que en caso de modificación solo fuera necesario reescribir parcialmente la capa afectada. Otra herramienta interesante desarrollada para este proyecto fue lo que se conocía como macrotransformations: una subrutina incorporada en ARCHIMODOS, ${ }^{13}$ en la que se agrupaban distintas operaciones formales de manera consecutiva (por ejemplo, traslación, rotación y escala) y que podían ser aplicadas al modelo produciendo una familia de deformaciones, sin una idea completamente acabada del resultado a obtener (Lynn 2013, 55).

Una vez que Yessios introducía las subrutinas en el ordenador, este efectuaba las operaciones formales y generaba un modelado alámbrico bidimensional del proyecto, algo que permitía mantener controlada la complejidad del modelo. Posteriormente, cuando el proyecto evolucionaba, se le añadía la tercera magnitud, permitiendo que las formas girasen y se movieran en los tres planos $X, Y$ y $Z$ : un proceso que, frecuentemente, requería pintar algunas de las caras de los objetos con el fin de ocultar las líneas en vista y acentuar la tridimensionalidad del modelo alámbrico. Del mismo modo, siguiendo un razonamiento fractal, las formas se escalaban en función de su dimensión raíz. Y si bien era posible ajustar la escala de una manera no uniforme, se optó por una escala uniforme: «Si había demasiada distorsión, ya no se podía reconocer el objeto» (Lynn 2013, 58).

A continuación, los resultados se imprimían en papel Mylar y eran enviados por mensajería privada al estudio de Eisenman. Cada mañana se recibía en las oficinas del arquitecto en Nueva York un paquete con los planos del proyecto actualizado.

Ya en el estudio, el resultado era fotocopiado, ampliando y reduciendo la escala de las formas, haciendo cálculos para encajar las partes, un proceso artesanal donde el proyecto era corregido a mano agregando anotaciones en rojo, redibujando 0 definiendo nuevas alternativas. $^{14}$

13.Muchas de estas subrutinas se integrarían definitivamente en sucesivas versiones de ARCHIMODOS, y pasarían después a formar parte de FormZ.

14.Para Joe Tanney, estos esquemas fueron apodados esquemas «klingons», en relación con la serie televisiva Star Trek: un conjunto de pequeñas formas geométricas desarrolladas bajo un proceso fractal (Lynn 2013, 72). 


\section{artnodes}

https://artnodes.uoc.edu

El autor y el intérprete: una mirada contemporánea al proyecto del Biocentro para la Universidad de Frankfurt de Peter Eisenman

Para este proceso, se trabajaba en un espacio cartesiano abstracto: una malla geométrica/numérica en la que se superponían, a modo de un palimpsesto analógico, los diferentes planos y secciones; a continuación, sobre la documentación se colocaba un papel transparente en donde se dibujaba a mano las nuevas modificaciones (Lynn 2014). Se trataba de un proceso que operaba por acumulación, superponiendo diferentes operaciones formales de traslación, rotación, escalado, recorte y extracción de la forma. Una evolución proyectual que iba «dejando sus rastros, sus huellas, su biografía generativa, que han de ser visibles y reconocibles» (García Sánchez 2018, 90). Layers que se superponían y crecían hacia arriba y hacia abajo, dejando vestigios de un código que prevalece en el objeto y queda plasmado en él. Se trata de un diseño conceptual bajo un proceso intelectual, con múltiples avances y retrocesos, en el que «su proceder no está orientado a la producción de un artefacto final formalmente puro, sino a la explicitación de ese proceso» (García Sánchez 2018, 86).

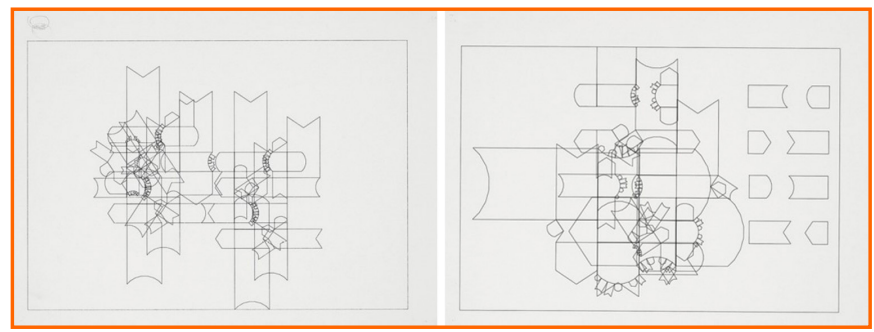

Figura 6. Investigación proyectual para el Centro de Investigaciones Biológicas Avanzadas, de la Universidad de Frankfurt, Alemania

Fuente: Lynn, Greg. Peter Eisenman, Biozentrum. Montreal: Canadian Centre for Architecture, 2014

Finalmente, estos nuevos dibujos serían traducidos al código alfanumérico, para ser dictados a Yessios, reiniciándose el ciclo una vez más (Lynn 2013, 54). Pese a lo rudimentario que pudiera parecernos este sistema de trabajo hoy en día, lo interesante de este proceso radicaba en la utilización del ordenador como una variable de aleatoriedad dentro del diseño. Conceptualmente, Eisenman «diseñaba los caminos a seguir», mientras que Yessios los interpretaba y elaboraba los procesos algorítmicos, dejando a los sistemas digitales la tarea de transitarlos y producir los resultados formales: un híbrido entre el diseño analógico y el digital.

\section{Conclusiones}

El proyecto del Biocentro para la Universidad de Frankfurt puede ser entendido como el predominio de una idea por encima del objeto físico, una reflexión propiamente lógica y cuyo sentimiento de placer parece ser alcanzado solo cuando la obra es traducida en concepto (Montaner 1993, 168). Tanto para el doctor Simón Marchán Fiz como para el arquitecto Josep María Montaner, en Eisenman «el modo de hacer se ha convertido en algo más importante que el artefacto», es decir, se ha desplazado la atención «desde la obra de arte como proceso acabado hacia el énfasis en el proceso de creación» (Montaner 1993, 168). Una mutación que llevará al espectador a ser quien deba «descubrir -en el sentido detectivesco del término- las huellas de un suceso, en suma: las pistas expresadas por el artista en el proceso» (García Sánchez 2018, 89).

De acuerdo con Greg Lynn, Eisenman no era consciente de que el proyecto del Biocentro estaba abriendo una nueva forma de observar y entender la arquitectura. Su búsqueda no era estética, sino que se hacía con un enfoque morfológico conceptual de la figura: se trataba de un proceso protoparamétrico, un procedimiento de codificación del proyecto bajo un pensamiento algorítmico racional, una visión lingüista de símbolos, no en el sentido de una gramática derridiana, sino desde el punto de vista de los fractales de Mandelbrot, en el que pudiera descubrirse las instrucciones que representaban la transformación y crecimiento de la forma $(2014,15)$.

A tal efecto, es interesante destacar que Eisenman estaba bosquejando lo que posteriormente se traduciría en un salto cuántico en el uso del ordenador en el campo del diseño, es decir, su utilización no como una herramienta de representación del espacio, sino como un instrumento de diseño: una circunstancia que convirtió al diseñador en el autor de un conjunto dado de reglas en lugar del autor de un diseño único (Lynn 2014, 15).

En este sentido, es conveniente destacar que la producción quedaba en manos externas, «el arquitecto no es el constructor, solo quien tiene la idea cuya generación se expresa en el proyecto ejecutado por un segundo actor» (García Sánchez 2018, 89). Y aquí es donde la crítica arquitectónica ha ubicado a la figura de Yessios, al cumplir las funciones de «intérprete» del proceso, traduciendo las ideas de Eisenman, primero a código y después a modelos digitales. Sin embargo, Yessios parece haber cumplido un rol más relevante del proceso de diseño del Biocentro; al crear los algoritmos y procedimientos que relacionaban las variables de evolución de la forma, también seleccionaba de un modo proyectual aquellas configuraciones que mejor se ajustaban a las soluciones deseadas. Trabajaba con bit de información que, como genes de un organismo biológico, se unían y vinculaban para permitir el crecimiento y la mutación del proyecto, mediante un diseño casuístico e impredecible que traducía las variables de tiempo y espacio adaptándolas a las condiciones particulares de un entorno binario complejo.

El ordenador era una máquina que potenciaba el desarrollo creativo hacia resultados indeterminados; no obstante, este requería de las capacidades de Yessios y de su equipo para adaptar y controlar esos procesos, que con frecuencia debían detenerse y readaptarse con nuevos parámetros.

Para Yessios, «lo paradójico del sistema es que muchos de los resultados obtenidos difícilmente hubieran sido posibles, sin la ayuda del ordenador y, sin embargo, éramos quienes le decíamos qué tenía que hacer» $(1987,170)$. En este sentido, agrega Yessios, refiriéndose a los planteamientos de Eisenman: «es probable que 


\section{artnodes}

https://artnodes.uoc.edu

El autor y el intérprete: una mirada contemporánea al proyecto del Biocentro para la Universidad de Frankfurt de Peter Eisenman

muchos de sus conceptos originales se perdieran o distorsionaran parcialmente [...] debido a que, cada diseñador utilizaba principalmente aquellas partes de las herramientas informáticas en las que había participado» $(1987,181)$.

Esencialmente, se trataba de un proceso complejo en el que hoy resulta difícil definir de forma precisa las fronteras que delimitaban el trabajo entre autor e intérprete. Sin embargo, de acuerdo con esta investigación, la actuación de Yessios parece superar ampliamente la misión de intérprete para entrar en el mundo de la creación, al diseñar procesos, a la vez que incorporaba y tomaba decisiones de cómo estos se empleaban. Yessios estaba influyendo directamente en el resultado final de la obra.

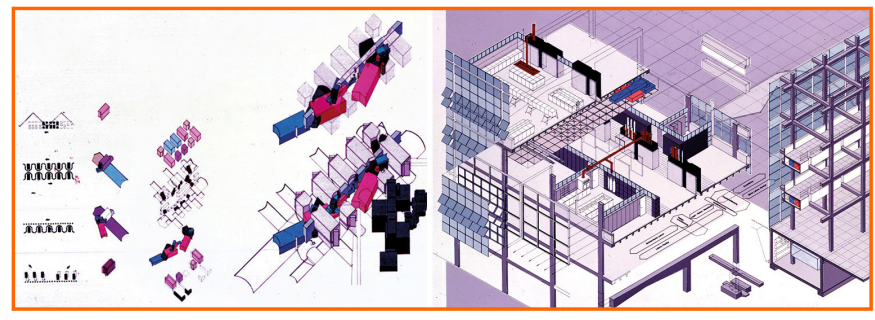

Figuras 7 y 8 . Evolución formal del proyecto para el Centro de Investigaciones Biológicas Avanzadas, de la Universidad de Frankfurt, Alemania

Fuente: Eisenman, Peter. «Biocenter». Consultado el 20 de julio de 2021, (1987). https:// eisenmanarchitects.com/Biocenter-1987

En una entrevista llevada a cabo en 2014, Eisenman le pregunta a Lynn, refiriéndose al proyecto del Biocentro, si creía que se podría haber dibujado el hilado y la iteración sin el ordenador. Lynn respondió que «La cuestión es si se podría haber llegado al proyecto del Biocentro sin un ordenador, sin el proceso [...] El ordenador iteraba por ti. Podías decir: Dame esto, lo otro o lo de más allá, y podía hacerlo» $(2014,15)$. Sin embargo, a través de la información expuesta en este artículo, en esta afirmación de Lynn existe un vacío, una ausencia, ya que entre el ordenador y los pedidos de Eisenman se encontraba la figura de Yessios, un coautor encargado no solo de diseñar las herramientas y algoritmos capaces de generar la forma, sino también de todos aquellos procesos que conducirían a la gestación y desarrollo del proyecto del Biocentro para la Universidad de Frankfurt.

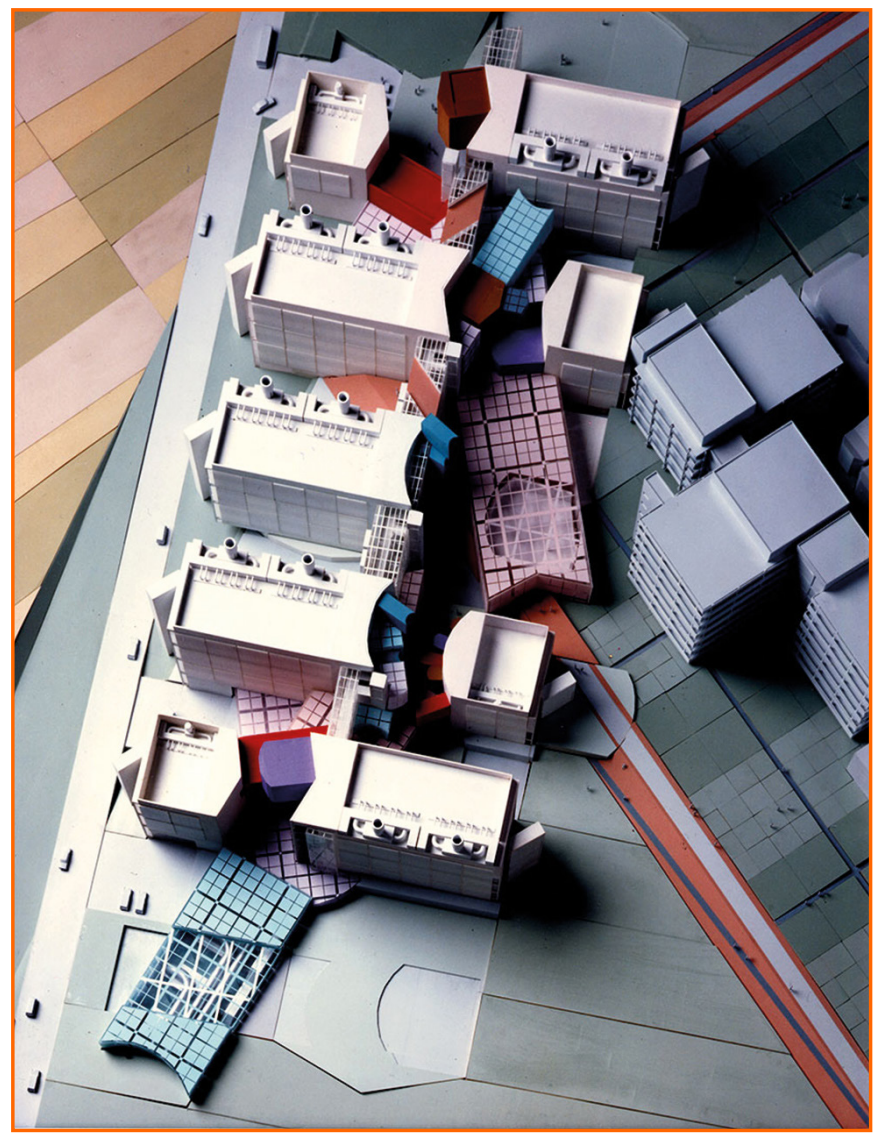

Figura 9. Maqueta final del proyecto para el Centro de Investigaciones Biológicas Avanzadas, de la Universidad de Frankfurt, Alemania

Fuente: Eisenman, Peter. «Biocenter». Consultado el 20 de julio de 2021, (1987). https:// eisenmanarchitects.com/Biocenter-1987

\section{Agradecimientos}

Un profundo agradecimiento al profesor Chris Yessios por su amable colaboración, así como también al profesor Daniel Cardozo Llach (Carnegie Mellon University) por la documentación facilitada. 


\section{Referencias bibliográficas}

CELADE. FORTRAN IV. Santiago de Chile: Centro Latinoamericano de Demografía, 1975.

Chomsky, Noam. Estructuras sintácticas. Madrid: Siglo XXI Editores, 1974.

Eisenman, Peter. «Biocenter». Consultado el 20 de julio de 2021, (1987). https://eisenmanarchitects.com/Biocenter-1987

García Sánchez, Rafael. «Concepto y proceso en Peter Eisenman y Sol LeWitt: la disolución de la forma». En: Liño. Revista Anual de Historia del Arte, no. 24, 85-94 (2018). D0l: https://doi.org/10.17811/ li.24.2018.85-94.

Graafland, Arie. Architectural Bodies, V.1. Rotterdam: Michael Speaks

Jormakka, Kari. «Generative processes». En: Basics Design Methods, 66-80. Berlin, Boston: Birkhäuser, 2017. D0l: https://doi. org/10.1515/9783035612387-009.

Lynn, Greg. Archaeology of the Digital: Peter Eisenman, Frank Gehry, Chuck Hoberman, Shoei Yoh. Montreal: Canadian Centre for Architecture, 2013.

Lynn, Greg. Peter Eisenman, Biozentrum. Montreal: Canadian Centre for Architecture, 2014.

Mandelbrot, Benoît. La geometría fractal de la naturaleza. Barcelona: Tusquets Editores, 1997.

Marchán Fiz, Simón. Del arte objetual al arte de concepto. Epílogo sobre la sensibilidad «Postmoderna». Madrid: Akal, 2009.

Moneo, Rafael. Inquietud teórica y estrategia proyectual en la obra de ocho arquitectos contemporáneos. Barcelona: Actar, 2004.

Montaner, Josep María. Después del movimiento moderno. Arquitectura de la segunda mitad del siglo XX. Barcelona: Gili, 1993.

Serraino, Pierluigi. History of Form ${ }^{\star} Z$. Basel: Birkhäuser, 2002.

Whiting, Sara. «Evidencia de cosas nunca vistas». En: Tras el rastro de Eisenman, Cynthia Davidson (eds.): 91-176. Madrid: Akal, 2006.
Yessios, Chris. «A Fractal Studio». Integrating Computers into the Architectural Curriculum [ACADIA Conference Proceedings] Raleigh (North Carolina / USA): 169-182, 1987.

Yessios, Chris. Archimodos. User's Manual. Ohio: The Ohio State University and International Business Machines Corporation, 1985.

\section{Índice de imágenes}

Figura 1 y 2. Representación artificial del ADN, utilizada por los científicos para explicar los procesos genéticos. Lynn, Greg. Peter Eisenman, Biozentrum. Montreal: Canadian Centre for Architecture, 2014.

Figura 3 y 4. Proceso de diseño utilizando geometrías fractales análogas a los procesos biológicos. Yessios, Chris. «A Fractal Studio». Integrating Computers into the Architectural Curriculum [ACADIA Conference Proceedings] Raleigh (North Carolina / USA): 179-180, 1987.

Figura 5 y 6 . Investigación proyectual para el Centro de Investigaciones Biológicas Avanzadas, de la Universidad de Frankfurt, Alemania. Lynn, Greg. Peter Eisenman, Biozentrum. Montreal: Canadian Centre for Architecture, 2014

Figura 7 y 8 . Evolución formal del proyecto para el Centro de Investigaciones Biológicas Avanzadas, de la Universidad de Frankfurt, Alemania. Eisenman, Peter. «Biocenter». Consultado el 20 de julio de 2021, (1987). https://eisenmanarchitects.com/Biocenter-1987.

Figura 9. Maqueta final del proyecto para el Centro de Investigaciones Biológicas Avanzadas, de la Universidad de Frankfurt, Alemania. Eisenman, Peter. «Biocenter». Consultado el 20 de julio de 2021, (1987). https://eisenmanarchitects.com/Biocenter-1987. 


\section{artnodes}

\section{CV}

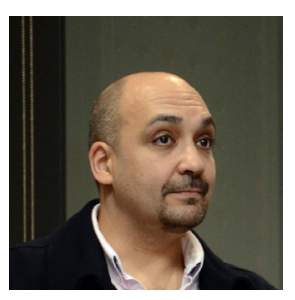

\section{Marcelo Fraile-Narváez}

Escuela Superior de Ingeniería y Tecnología

Universidad Internacional de La Rioja

marcelo.fraile@unir.net

Doctor en Arquitectura por la Universidad de Buenos Aires. Especialista en diseño biodigital. Máster en Conservación y Rehabilitación del Patrimonio Arquitectónico (UNC), arquitecto (UNT). Como fundador de la revista y el colectivo de diseño TRP21, mi línea de investigación se enmarca en un campo interdisciplinario: una relación entre las nuevas tecnologías y la biología, un nuevo campo de experimentación en el diseño contemporáneo. Miembro de los grupos de investigación Diseño, Sociedad, Educación y Arte (UNIR); Museum I+D+C. Laboratorio de Cultura Digital y Museografía Hipermedia (UCM), y de los proyectos Diseño de infografías científicas en el aula a través de herramientas web 3.0 y recursos en abierto (UCM) y La utilidad de las infografías como recurso pedagógico en el proceso de enseñanza-aprendizaje (UIRPPEA-UNED). Con veinticinco años como profesor universitario, ha impartido cursos de grado y posgrado en diferentes instituciones de América y de España. Coordinador del proyecto Biomímesis, MediaLab Prado. Autor de artículos sobre crítica arquitectónica, la historia y el diseño biodigital.

https://marcelofrailenarvaez.wordpress.com/ https://orcid.org/0000-0002-9321-4512 